\title{
Nanotoxicology : An Emerging Discipline
}

\author{
Sachin Kumar Jain*, Y. P. Sahni, Neetu Rajput and Vidhi Gautam \\ Department of Veterinary Pharmacology and Toxicology, \\ College of Veterinary Science and Animal Husbandry, \\ Jabalpur - 482001 (M. P.), INDIA. \\ * Corresponding author
}

\begin{abstract}
Nanotoxicology refers to the study of the interactions of nanostructures with biological systems with an emphasis on elucidating the relationship between the physical and chemical properties of nanostructures with induction of toxic biological responses. Nanotoxicology is aimed at providing information on the potential toxicological effects, risk assessment and safety evaluation of nanostructured materials on human health. Nanoparticles present possible dangers, both medically and environmentally. They are also able to pass through cell membranes in organisms and their interactions with biological systems are relatively unknown. Animal studies have shown that nanoparticles can penetrate cells and tissues, move through the body and brain and cause biochemical damage. The greater chemical reactivity of nanomaterials result in increased production of reactive oxygen species which may contribute to similar patterns of cell injury and alterations at the molecular level by initiation, propagation and autocatalytic chain reactions. Intracellular signaling activation and inactivation of enzymes, stimulation, secretion and release of pro-inflammatory cytokines, chemokines and nuclear factor activation and alteration are also common events.
\end{abstract}

Keywords: Nanotoxicology, Nanomaterials, Reactive oxygen species.

\section{I ntroduction}

A wide variety of nano-structured materials are now used in commodities, pharmaceutics, cosmetics, biomedical products, and industries. While nanoscale materials possess more novel and unique physicochemical properties than bulk materials, they also have an unpredictable impact on human health. The entrance into and interaction of nanomaterials in the human body have generated intense scientific curiosity, attracting much attention as well as increasing concern from the public, nanomaterialbased industries, academia, and governments worldwide. (Dreher, 2004)

The development of technology enables the reduction of material size in science. The use of particle reduction in size from micro to nanoscale not only provides benefits to diverse scientific fields but also poses potential risks to humans and the environment. For the successful application of nanomaterials in bioscience, it is essential to understand the biological fate and potential toxicity of nanoparticles. (Colvin, 2003).

Nanotoxicology refers to the study of the interactions of nanostructures with biological systems with an emphasis on elucidating the relationship between the physical and chemical properties (e.g. size, shape, surface chemistry, composition, and aggregation) of nanostructures with induction of toxic biological responses.

Nanotoxicology is aimed at providing information on the potential toxicological effects, risk assessment, and safety evaluation of nano-structured materials on human health for a wide audience, including those working in the fields of nanoscience and nanotechnology, biology, toxicology, chemistry, chemical biology, medical sciences, public health sciences, standardization of nanotechnology (nanosafety standards) as well as for policy makers developing regulatory frameworks in government departments and nanotechnology industries /enterprises. Nanotoxicology, dealing with the potential impact of nanoparticles on human and animal health and environment, evolved as a new field of research (Yezhelyez et al., 2006).

\section{Classification of Nanoparticles}

( I) Combustion-derived nanoparticles (CDNP):

Combustion-derived nanoparticles present a diverse group of materials which gain commonality because of their origin in combustion processes and their demonstrated toxicity in various models. Common examples are diesel exhaust particles, welding fumes, fly-ash. 
Nanotoxicology : An Emerging Discipline

Table-1: CDNP considered here are described in the following table along with their salient effects in humans and animals

\begin{tabular}{|c|c|c|}
\hline CDNP & Origin & Reported Health effects \\
\hline Diesel exhaust particles & Combustion of diesel oil & Inflammation, fibrosis,cancer \\
\hline Welding fume & Welding processes & Inflammation; translocation of metals to the brain \\
\hline Nanoparticle Carbon black & Combustion of heavy fuel oil & $\begin{array}{l}\text { Inflammation, lung cancer; translocation of particles } \\
\text { to the brain }\end{array}$ \\
\hline
\end{tabular}

(Salata, 2004)

CDNP Origin Reported Health effects Diesel exhaust particles Combustion of diesel oil Inflammation, fibrosis, cancer Welding fume Welding processes Inflammation; translocation of metals to the brain Fly-ash Combustion of coal or oil inflammation Nanoparticle Carbon black Combustion of heavy fuel oil Inflammation, lung cancer; translocation of particles to the brain (Salata, 2004)

\section{(ii) Manufactured derived nanoparticles:}

Manufactured nanoparticles display physicochemical characteristics including electrical, thermal, mechanical, and imaging properties that are highly desirable for applications in commercial, medical and environmental sectors (Masciangioli and Zhang, 2003).

(iii) Naturally occuring nanoparticles:

Biologically based or naturally occuring molecules that are found inside organism. For example; biogenic magnetite is a naturally occuring nanosized particle that occurs in many species ranging from bacteria to protozoa, to animals. Biogenic magnetite is even been found in brain and has been associated with neuro degenerative diseases (Antunes et. al, 2007).

\section{Nanomaterials in the environment}

During the manufacture, transport, use and disposal of nanomaterials and those products containing nanomaterials, the release of these materials into the environment is inevitable. As the use of nanomaterials increases, so too will their presence in the environment. The details are given in the Table - 3 .

\section{Mechanism of action of nanoparticles}

The behavior of nanoparticles is a function of their size, shape and surface reactivity with the surrounding tissue.

The smaller a particle, the greater it's surface area to volume ratio and the higher its chemical reactivity and biological activity. The greater chemical reactivity of nanomaterials results in increased production of reactive oxygen species (ROS).

ROS production has been found in a diverse range of nanomaterials including carbon nanotubes and nanoparticle metal oxides. ROS and free radical production is one of the primary mechanisms which may result in oxidative stress, inflammation and consequent damage to proteins, membranes and DNA.

Both in vivo and in vitro, Nanosized particles of various chemistries have been shown to create reactive oxygen species (ROS). ROS production has been found in Nps as diverse as C60 fullerenes, SWNTs, quantum dots, and UFPs, especially under concomitant exposure to light, UV, or transition metals (Brown et al., 2000, 2001; Derfus et al., 2004; Joo et al., 2004; Oberdorster E, 2004a). It has been demonstrated that NSPs of various sizes and various chemical compositions preferentially mobilize to mitochondria (Foley et al, 2002; Rodoslav et al., 2003). Because mitochondria are redox active organelles, there is a likelihood of altering ROS production and thereby overloading or interfering with antioxidant defenses.

Some of the antioxidant defense systems that occur in animals, and possible areas where NSPs may create oxyradicals. The exact mechanism by which each of these diverse NPs cause ROS is not yet fully understood, but suggested mechanisms include:

Table-2 : Common Nanomaterials and Their Uses

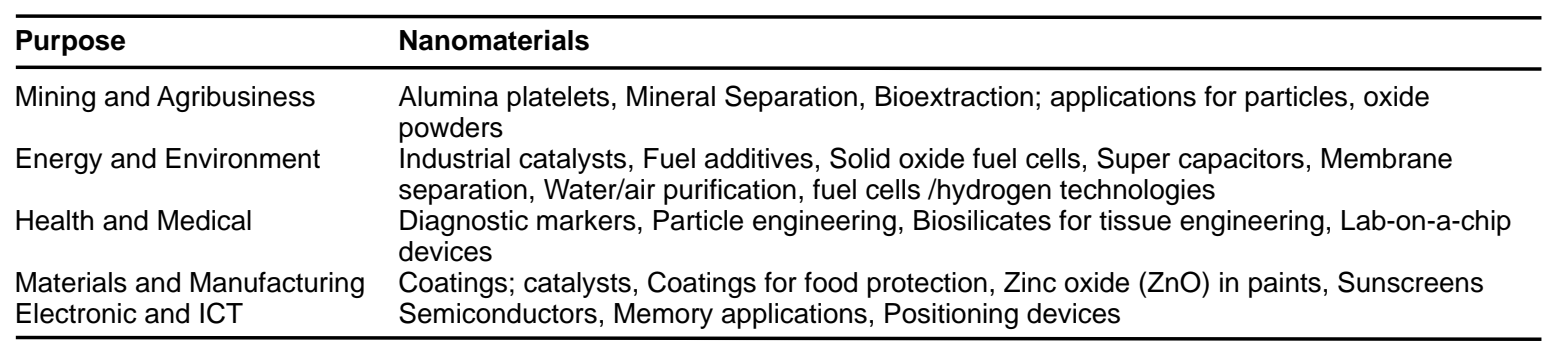


Nanotoxicology : An Emerging Discipline

Table-3: Environmental release of Nanomaterials.

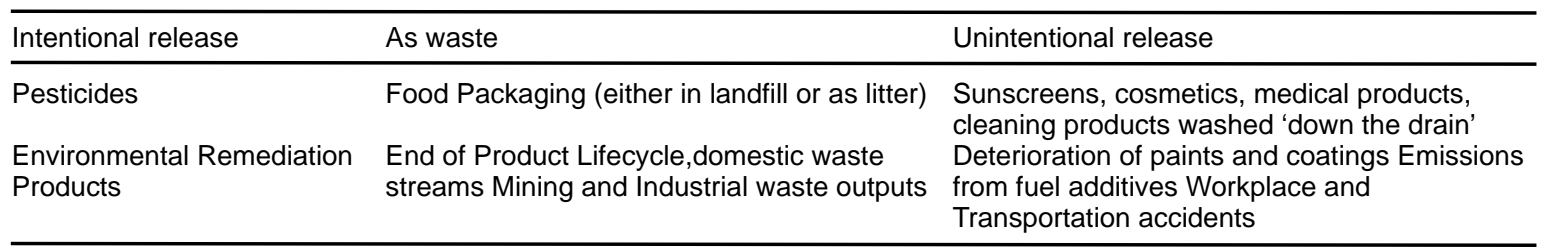

(J orgensen et al., 2005)

a) photo excitation of fullerenes and SWNTs, causing intersystem crossing to create free electrons;

b) metabolism of NPs to create redox active intermediates, especially if metabolism is via cytochrome P450s; and

c) inflammation responses in vivo that may cause oxyradical release by macrophages. Other mechanisms will likely emerge as studies on NP toxicity continue.

\section{Translocation of Nanoparticles}

\section{Blood Stream}

Nanoparticles could avoid normal phagocytic defences in the respiratory system and gain access to the systemic circulation or even to the central nervous system. Once inhaled and deposited, nanoparticles can translocate to extra pulmonary sites and reach other target organs by different mechanisms. The first mechanism involves passing of nanoparticles across epithelia of the respiratory tract into the interstitium and access to the blood stream directly or via lymphatic pathways, resulting in systemic distribution of nanoparticles. Berry et al (1977) observed the presence of nanoparticles in rat platelets after intratracheal instillation of colloidal gold particles (30 $\mathrm{nm})$. Once nanoparticles are translocated into the blood stream they could induce adverse biological effects (Oberdorster et al., 2004).

\section{Central nervous system}

The translocation of nanoparticles to central nervous system takes place as a result of systemic distribution. The other mechanism involves the uptake of nanoparticles by sensory nerve endings embedded in airway epithelia, followed by axonal translocation to ganglionic and CNS structures. In addition, nanoparticles can be taken up by the nerve endings of the olfactory bulb and translocated to the CNS (Letham, 2003).

\section{Gastrointestinal tract}

Nanosize particle cleared from the respiratory tract via the mucociliary escalator can subsequently be ingested into the gastrointestinal tract. Alternatively, nanomaterials can be ingested directly, for example, if contained in food or water or if used as drugs or drug delivery devices. Only a few studies have investigated that if nanosized particles contained in food or water are used as drugs or drug delivery devices, they can be ingested directly and pass through the gastrointestinal tract and are eliminated rapidly (Hoet et al., 2004).

\section{Skin}

A potentially important uptake route is through dermal exposure. Broken skin represents a readily available portal of entry even for larger particles. Subsequent translocation of nano size particle beyond lymph nodes to the blood circulation is likely to occur.

\section{Toxicity of Nanoparticles}

Nanoparticles present possible dangers, both medically and environmentally. Most of these are due to the high surface to volume ratio, which can make the particles very reactive or catalytic. (Wiesner et al., 2006). They are also able to pass through cell membranes in organisms, and their interactions with biological systems are relatively unknown.

According to the San Francisco Chronicle, "Animal studies have shown that some nanoparticles can penetrate cells and tissues, move through the body and brain and cause biochemical damage. Diesel nanoparticles have been found to damage the cardiovascular system in a mouse model (Royal Society and Royal Academy of Engineering, 2004).

Lungs : One of the most important portals of entry and organ target for nanoparticles is the respiratory system. It is well known that lungs are easily exposed to atmospheric pollutants and many other products of thermo-degradation. In this regard, combustionderived nanoparticles have been largely studied as a possible etiologic factor for several adverse health effects, including exacerbations of airways disease as well as deaths and hospitalization from cardiovascular disease (Donaldson, 2005).

Researchers in the University of Texas in the United States found that carbon nanotubes squirted into the trachea of mice caused serious inflammation of the lungs and granulomas (tumour-like nodules of 
bloated white blood cells in the lining of the lungs), and five of the nine mice treated with the higher dose died (Oberdorster et al., 2005). In yet another similar experiment in Tottori University, Japan, researchers showed that within a minute of contacting the mice's tiniest airways, carbon nanotubes began to burrow through gaps between the surface lining cells and into the blood capillaries, where the negatively charged nanoparticles latched onto the normally positively charged red blood cells surface, thereby potentially causing the red blood cells to clump and the blood to clot (Lam et. al., 2004).

Heart : The Institute of Occupational Medicine U.S. shows that various nanoparticles when introduced into the bloodstream can cause heart tissue damage. This arises through oxidative stress and inflammation leading to apoptosis and necrosis (Yoshifumi, 2002).

Some reports show that nanoparticles particularly Calcium nanoparticles produce heart attacks. In an experiment, scientist injected lab animals' artery walls with these Calcium nanoparticles and the walls became inflamed. Inflammatory response is a first step towards hardening (Oberdorster et al., 1995)

Brain: In some of the experiments conducted by using mice brain cell cultures found that introducing nanoscale titanium dioxide caused oxidative stress in neurons. This is of concern as the oxidative stress of neuron cells has been linked with neurodegenerative diseases.

A number of pathologies, including hypertension and allergic encephalomyelitis, however, have been associated with increased permeability of the blood brain barrier to Nanoparticles in experimental set ups. The nanoparticle surface charge has been shown to alter blood-brain integrity and need consideration as to their role in brain toxicity and brain distribution.

Nanoparticles have been shown to induce the production of reactive oxygen species and oxidative stress has been implicated in the pathogenesis of neurodegenerative diseases such as Parkinson's and Alzheimer's (Dagani, 2003).

Gastrointestinal tract: Another portal of nanoparticles entry in the body is GI tract. Nanoparticles can be ingested into the gut by many ways, such as; nanoparticles can be ingested directly from the food, water and drugs (Hoet et al., 2004).

Recently, it has been shown that $\mathrm{Cu}$ nanoparticles administered via oral gavage can induce an inflammatory response leading to bowel disease that includes both ulcerative colitis and Crohn's disease
(CD) is an inflammatory chronic condition whose aetiology remains still unclear (Chen et al., 2006). It has been shown that a diet low in $\mathrm{Ca}++$ and exogenous microparticles alleviated the symptoms of human CD with a significant improvement in the $\mathrm{CD}$ activity index (Jani et al., 1994). These results are particularly relevant to $\mathrm{CD}$ as an abnormal intestinal permeability has been found in this disease (Szentkuti, 1997).

Liver: The effect of Quantum dots on liver cells was examined by Roco (2003) in the University of California. Researchers found that cadmium-selenium quantum dots were acutely toxic to rat liver cell cultures at doses similar to that expected for human exposure.

The reticulo-endothelial system located in the liver is exposed to all nanoparticles absorbed from the gastrointestinal tract (GIT) into the cardiovascular system, since all blood exiting the GIT does so in the hepatic portal vein that directly diffuses through the liver. Low toxicity Nanoparticles such as carbon black and polystyrene stimulate the macrophages via reactive oxygen species and calcium signalling, to make pro inflammatory cytokines such as tumour necrosis factor alpha (Chad and Andrew, 2000). Oxidative stress is known to inhibit hepatocyte function and bile formation, while pro-inflammatory cytokines are also associated with the pathology of liver disease (Yoon et al., 2005).

Skin: Nanomaterials such as nanoscale titanium dioxide and zinc oxide have widespread use in ointments and creams; therefore, an understanding of their effect on skin is essential in assessing the risk to both human and animals. The widespread environmental presence of other manufactured nanomaterials could also lead to skin exposure.

Initial studies showed that certain nanomaterials can cause damage to skin cells as well as pass through the epidermal (outermost) layer of skin into deeper layers and subsequently into the body and bloodstream.

Due to their small size, nanomaterials also have the potential to be absorbed through the skin and enter the blood stream or lymphatic system (M.I.T., 2007). Nanoparticles can also be taken up by lymphatic nodes at skin level, translocating to the blood stream via lymphatic pathways (Kim et al., 2004).

Plants: Nanoparticles could have a negative effect on plant growth and have the ability to cause injury to plants. A new study examined the effects of five types of nanoparticles on seed germination and root growth of six higher plant species and observed that several types of the particles had significant inhibition on seed 
germination and root growth of the six plants. If confirmed, these results are significant in terms of use and disposal of engineered nanoparticles (Yang and Watts, 2005). Scientific reports showed that aluminum oxide nanoparticles, commonly found in everything from drugs to environment, can stunt root growth in plant species like corn, cucumber, soybean, carrot and cabbage.

\section{Detection of Nanoparticles}

There is a great problem in the detection of nanoparticles, due to the fact that,

- $\quad$ they do not localize in a specific area and diffuse quickly thereby allowing them to afflict many people in a short duration.

- due to their high surface area and increased reactivity.

- they are able to aggregate and form agglomerates which again cannot be detected easily.

Therefore, there is a need for an ideal detection system which would be cost effective, be able to perform direct sampling, provide information regarding airborne NPs, detect the NPs as aggregates, and be specialized for the specific NPs within that work environment.

\section{Conclusion}

Nanotoxicology has emerged as a new branch of toxicology for studying undesirable effects of nanoparticles. Despite an overall lack of scientific research into the risks of nanomaterials, initial studies pointed to the high toxicity of some nanomaterials. Scientists are unanimous in asserting that there are still many gaps in the understanding of the risks posed to human health and the environment.

The development of engineered nanoparticles with substantial biomedical significance has posed new opportunities and challenges for pharmacology and therapeutics. Nanomaterials and nanoparticles are likely to be cornerstones of innovative nanomedical devices to be used for drug discovery and delivery, discovery of biomarkers and molecular diagnostics. Development of novel nanoparticles used in pharmacology, therapeutics and diagnostics must proceed in tandem with assessment of toxicological and environmental side effects of these particles.

\section{References}

1. Antunes, M.B. Bowler R. and Doty R L. (2007) Olfactory function. Neurology, 69:1278-1284.

2. Berry J.P., Arnoux B., Stanislas G., Galle P. and Chretien J. (1977) A microanalytic study of particles transport across the alveoli: role of blood platelets.
Biomedicine 27: 354-357.

3. Chad, A. M. and Andrew, T. (2000). Semiconductors meet biology. Nature 405: 626-627.

4. Chen Z, Meng H, Xing G, Chen C, Zhao Y and Jia G (2006). Acute toxicological effects of copper nanoparticles in vivo. Toxicol Lett 163 (2):109-120.

5. Colvin, V. L. (2003). The potential environmental impact of engineered nanoparticles. Nat. Biotechnol. 21: 1166-1170.

6. Dagani, R. (2003) Nanomaterials: Safe or Unsafe? Chem. \& Eng. News 30-33. http://www.cenonline.org or http://pubs.acs.org.

7. Derfus A.M., Chan W.C.W. and Bhatia S.N. (2004) Probing the cytotoxicity of semiconductor quantum dots. Nano. Lett. 4(1):11-18.

8. Donaldson A. (2005). Ultrafine particles: Mechanisms of lung injury. Philosophical Transactions of the Royal Society A: Mathematical, Physical and Engineering Sciences 358 p 2741.

9. Dreher, K. (2004) Health and Environmental Impact of Nanotechnology: Toxicological Assessment of Manufactured Nanoparticles. Toxicol. Sci., 77 (1):3-5.

10. Foley S, Crowley C., Smaihi M, Bonfils C., Erlanger B.F., Seta P., et al., (2002) Cellular localisation of a water-soluble fullerene derivative. Biochem. Biophys. Res. Commun. 294:116-119.

11. Hoet P, I Bruske-Hohlfeld and Salata O (2004) Nanoparticles - known and unknown health risks. J Nanobiotechnol 2: 12. http://www.itas.fzk.de/tatup/ 051/joua05a.htm

12. Jani, P.U., McCarthy, D.E. and Florence, A.T. (1994) Titanium dioxide (rutile) particle uptake from the rat GI tract and translocation to systemic organs after oral administration. Int. J. Pharm. 105: 157-168.

13. Joo S.H., Feitz A.J. and Waite T.D. (2004) Oxidative degradation of the carbothioate herbicide, molinate, using nanoscale zero-valent iron. Environ Sci Technol 38: 2242-2247.

14. Jorgensen M S., Pedersen T.T., Felch M., and Jorgensen U. (2005) Green Technology Foresight about Environmentally Friendly Products and Materials, Danish Environment Protection Agency p 196.

15. Kim S, Lim Y.T., Soltesz E.G., De Grand A.M. Lee J. and Nakayama A. (2004). Near-infrared fluorescent type II quantum dots for sentinel lymph node mapping. Nat Biotechnol 22: 93-97.

16. Lam H., K. Poon, T. Burd (2004) Pulmonary Toxicity of Single-Wall Carbon Nanotubes in Mice 7 and 90 Days after Intratracheal Instillation. Toxicological Sciences 77: 126.

17. Letham. L.(2003). Thinking about issues associated with nanotechnology. News From the Bottom, 1(2): 116.

18. Li N., Sioutas C., Cho A., Schmitz D., Misra C., Sempf J., et al., (2003). Ultrafine particulate pollutants induce oxidative stress and mitochondrial damage. Environ Health Perspect 111:455-460. 
19. Masciangioli,T. andZhang W.X.(2003).Environmental Technologies and the Nanoscale. American Chemical Society. Envrionmental Science \& Techn. 102A - 108A.

20. Oberdörster E. (2004a). Manufactured nanomaterials (fullerenes, C60) induce oxidative stress in brain of juvenile largemouth bass. Environ Health Perspect 112: 1058-1062.

21. Oberdorster G., Gelein R. M., Ferin J. and Weiss B. (1995) Association of particulate air pollution and acute mortality: involvement of ultrafine particles? Inhal. Toxicol. 7:111-124.

22. Oberdorster, G., Oberdorster, E. and Oberdorster, J. (2005) Nanotoxicology: an emerging discipline evolving from studies of ultrafine particles. Nanotoxicology 113:823-839.

23. Oberdorster, G.; Sharp, Z.; Atudonrei, V.; Elder, A.; Gelein, R.; Kreyling, W. and Cox, C. (2004) Translocation of Inhaled Ultrafine Particles to the Brain. Inhalation Toxicol., 16: 453-459. 